\title{
Influence of Fibrotic Tissue Arrangement on Intracardiac Electrograms During Persistent Atrial Fibrillation
}

\author{
Jorge Sánchez ${ }^{1,2}$, Mark Nothstein ${ }^{1}$, Laura Unger ${ }^{1}$, Javier Saiz ${ }^{2}$, Beatriz Trénor ${ }^{2}$, Olaf Dössel ${ }^{1}$, Axel \\ Loewe $^{1}$ \\ ${ }^{1}$ Institue of Biomedical Engineering, Karlsruhe Institute of Technology (KIT), Karlsruhe, Germany \\ ${ }^{2}$ Centro de Investigación e Innovación en Bioingeniería $\left(\mathrm{Ci}^{2} \mathrm{~B}\right)$, Universitàt Politècnica de València, \\ Valencia, Spain
}

\begin{abstract}
Under persistent atrial fibrillation (peAF), cardiac tissue experiences electrophysiological and structural remodeling. Fibrosis in the atrial tissue has an important impact on the myocyte action potential and its propagation. The objective of this work is to explore the effect of heterogeneities present in the fibrotic tissue and their impact on the intracardiac electrogram (EGM). Human atrial myocyte and fibroblast electrophysiology was simulated using mathematical models proposed by Koivumäki et al. to represent electrical remodeling under peAF and the paracrine effect of the transforming grow factor $\beta 1$ (TGF- $\beta 1) .2 D$ tissue simulations were computed varying the density of fibrosis $(10 \%, 20 \%$ and $40 \%)$, myofibroblasts and collagen were randomly distributed with different ratios $(0 \%-100 \%, 50 \%-50 \%$ and $100 \%$ $0 \%)$. Results show that increasing the fibrosis density changes the re-entry dynamics from functional to anatomical due to a block in conduction in regions with high fibrosis density (40\%). EGM morphology was affected by different ratios of myofibroblasts-collagen. For low myofibroblast densities (below 50\%) the duration of active segments was shorter compared to higher myofibroblasts densities (above 50\%). Our results show that fibrosis heterogeneities can alter the dynamics of the re-entry and the morphology of the EGM.
\end{abstract}

\section{Introduction}

Atrial fibrillation (AF) is the most common cardiac arrhythmia. Fibrosis is a complex phenomenon involving different actors, such as myofibroblast proliferation, collagen deposition, inflammatory cytokines, among others, that may alter the action potential (AP) and its propagation. Intracardiac electrograms (EGMs) are of great clinical interest and recorded during electrophysiological studies. EGM fractionation can be caused by focal sources or reentrant mechanisms involved in arrhythmia maintenance, as well as by passive mechanisms such as wave collision, conduction slowing or block, wavebreaks and fibrosis.

Computational simulations of tissue electrophysiology give the opportunity to study the effect of heterogeneities in the atrial substrate in detail. There are different approaches to simulate fibrotic regions. Simulating nonconductive elements [1], ionic remodeling due to paracrine effect of TGF- $\beta 1$ [2], and similar approaches using ionic models which describe the electrophysiology of fibroblasts [1], [3]. However, each methodology to simulate fibrotic tissue is different and may alter the dynamics of the arrhythmia. Additionally, the correlation between EGM morphology, substrate remodeling and fibrosis is still unclear.

In this study we investigate different ways of modeling atrial fibrotic tissue including myofibroblasts, paracrine effect of TGF- $\beta 1$ and collagen deposition. We varied the density of fibrosis and the density ratio between collagen and myofibroblasts. We also analyzed bipolar EGMs calculated from our simulations and compared them with fibrosis distribution.

\section{Methods}

\subsection{Myocyte electrophysiological models}

The human atrial action potential (AP) model by Koivumäki et al. [4] was used to simulate atrial APs. To reproduce the tissue electrophysiology of different anatomical regions of the atrium, we modified the conductance of five ionic currents: transient outward $\mathrm{K}^{+}$ current $\left(\mathrm{I}_{\mathrm{t}}\right)$, potassium rapid current $\left(\mathrm{I}_{\mathrm{Kr}}\right)$, potassium slow current $\left(\mathrm{I}_{\mathrm{Ks}}\right)$, time independent $\mathrm{K}^{+}$current $\left(\mathrm{I}_{\mathrm{K} 1}\right)$, and $\mathrm{L}$ type $\mathrm{Ca}^{2+}$ current (I $\mathrm{I}_{\mathrm{CaL}}$ ) as proposed in previous simulation studies [5]. The maximum conductance of these currents were modified according to experimental values to reproduce the AP waveform of the posterior wall in the left atrium (LA). 


\subsection{Ionic remodeling}

AF electrical remodeling was introduced by modifying ion channel conductances for $\mathrm{I}_{\mathrm{CaL}}, \mathrm{I}_{\mathrm{to}}, \mathrm{I}_{\mathrm{K} 1}$, sustained outward $\mathrm{K}^{+}$current $\left(\mathrm{I}_{\text {sus }}\right), \mathrm{Na}^{+} / \mathrm{Ca}^{2+}$ exchanger (NCX), sarcoplasmic reticulum $\mathrm{Ca}^{2+}$ ATPase (SERCA) pump, and ryanodine receptors (RyR), and specific parameters of the calcium handling, such as phospholamban (PLB), sarcolipin (SLN), and the baseline phosphorylation (phos). Dilation was also modeled as described in Koivumäki et al. [6]. TGF- $\beta 1$ paracrine remodeling was represented by modifying ion channel conductances, $50 \%$ reduction in $\mathrm{I}_{\mathrm{K} 1}, 50 \%$ reduction $\mathrm{I}_{\mathrm{CaL}}$, and $40 \%$ reduction in sodium current $\mathrm{I}_{\mathrm{Na}}[2]$.

\subsection{Myofibroblast electrophysiological model}

Myofibroblast electrophysiology was represented using the Koivumäki et al. [7] model. The membrane sodium current was updated according to recent experimental results [8]. Additionally, uncoupled fibroblast resting membrane potential (RMPf) was set to $-26 \mathrm{mV}$, as suggested by experimental data [8], [9]. These values were obtained by shifting the gating variable for the time dependent potassium current as in previous simulation studies [3], [10]. Furthermore, the value of the fibroblast membrane capacitance $(\mathrm{Cmf})$ were varied within experimental ranges $(50.4 \mathrm{pF})$ and set a value of conductance for the gap junction of $0.5 \mathrm{nS}$ was set [8].

\subsection{Tissue simulations}

To simulate the electrical propagation of the AP, the reaction-diffusion equation (1) of the monodomain formulation was used:

$$
\nabla \cdot\left(D \nabla V_{m}\right)=C_{m} \frac{\mathrm{d} V_{m}}{\mathrm{dt}}+I_{\text {ion }}(1)
$$

where $\mathrm{D}$ is the diffusion coefficient, $\mathrm{V}$ is the transmembrane voltage, $C_{m}$ is the membrane capacitance, and $\mathrm{I}_{\text {ion }}$ stands for ionic currents through the membrane.

Diffusion coefficients (Dm) were calculated and adjusted to achieve physiological CVs, yielding a Dm of $3.84 \mathrm{~cm}^{2} / \mathrm{s}$ in the LA $(\mathrm{CV}=70 \mathrm{~cm} / \mathrm{s})$. An anisotropy ratio of 2.86:1 was considered in the LA [5]. Under peAF, the diffusion coefficient was reduced by $50 \%$ to reproduce gap junction remodeling [1].

Two-dimensional (2D) meshes, representing cardiac tissues for LA were built with a central fibrotic region of $2 \mathrm{~cm}$ diameter with different three different densities $(10 \%, 20 \%$ and $40 \%)$ uniformly and randomly distributed. Elements inside this region could be myocytes affected by peAF and TGF- $\beta 1$ ionic remodeled, myofibroblasts or collagen. Myofibroblasts and collagen were distributed with different ratios $(0 \%-100 \%, 50 \%$ $50 \%$ and $100 \%-0 \%$ correspondingly). The tissue grid had $256 \times 256$ elements with a spatial resolution of $200 \mu \mathrm{m}$ (Figure 1, panel A).

To study the effect of fibrosis heterogeneity on EGMs during a re-entry, reentrant activity was generated using a cross-field (S1-S2) stimulation protocol. S1 stimuli consisted of 10 pulses applied to the left border of the computational mesh to stabilize the tissue and S2 was applied to the left bottom corner of the tissue.

Surface pseudo-unipolar EGM were calculated using the infinity volume conductor approximation (equation 2):

$$
\Phi_{e}(r)=-\frac{1}{4 \pi} \frac{\sigma_{i}}{\sigma_{e}} \iiint \nabla V_{m}\left(r^{\prime}\right) \cdot \nabla\left[\frac{1}{r^{\prime}-r}\right] d v(2)
$$

where is $\sigma_{i}$ the intracellular conductivity, $\sigma_{e}$ the extracellular conductivity, $\nabla V_{m}\left(r^{\prime}\right)$ is the spatial gradient of the transmembrane voltage and $\mathrm{r}$ is the distance from the source point to the measuring point. Bipolar EGMs were calculated from two unipolar EGM with a spacing of $1 \mathrm{~mm}$ (Figure 1, panel B). The criteria for fractionation were based on the duration of the active segment of the signal using the Nonlinear Energy Operator algorithm described by Schilling et al. [11] and the Shannon Entropy. Signal with peak to peak values below $0.5 \mathrm{mV}$ were not considered for the calculations.

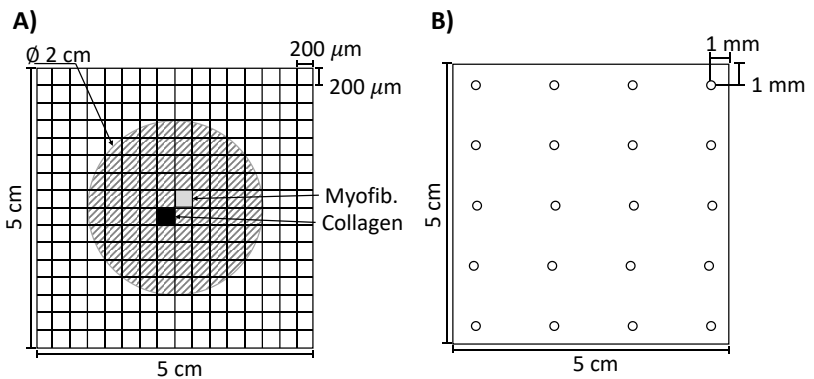

Figure 1. A) 2D tissue with uniformly distributed random fibroblasts density $(10 \%, 20 \%, 40 \%)$ in a defined region of $2 \mathrm{~cm}$. Within the region, myofibroblasts and collagen randomly distributed with different ratios $(0 \%-100 \%$, $25 \%-75 \%, 50 \%-50 \%, 75 \%-25 \%$ and $100 \%-0 \%)$. B) Spatial distribution of electrodes on the 2D tissue.

\section{Results}

Figure 2 shows the AP of a single myocyte under physiological conditions, electrically remodeled due to peAF and under the paracrine effect of the TGF- $\beta 1$. Under physiological conditions $\mathrm{APD}_{90}$ for LA myocytes was 214 ms. Under peAF electrical remodeling $\mathrm{APD}_{90}$ shortened to $139.8 \mathrm{~ms}$. The RMP dropped from $-75 \mathrm{mV}$ to $-79 \mathrm{mV}$ and $\left(\mathrm{dV}_{\mathrm{m}} / \mathrm{dt}\right)_{\max }$ increased from $163 \mathrm{~V} / \mathrm{s}$ to 168 $\mathrm{V} / \mathrm{s}$. In contrast, myocytes remodeled due to peAF and 
TGF $\beta-1$ had an $\mathrm{APD}_{90}$ of $198.1 \mathrm{~ms}$, RMP of $76.16 \mathrm{mV}$ and $\mathrm{a}\left(\mathrm{dV}_{\mathrm{m}} / \mathrm{dt}\right)_{\max }$ of $70 \mathrm{~V} / \mathrm{s}$.

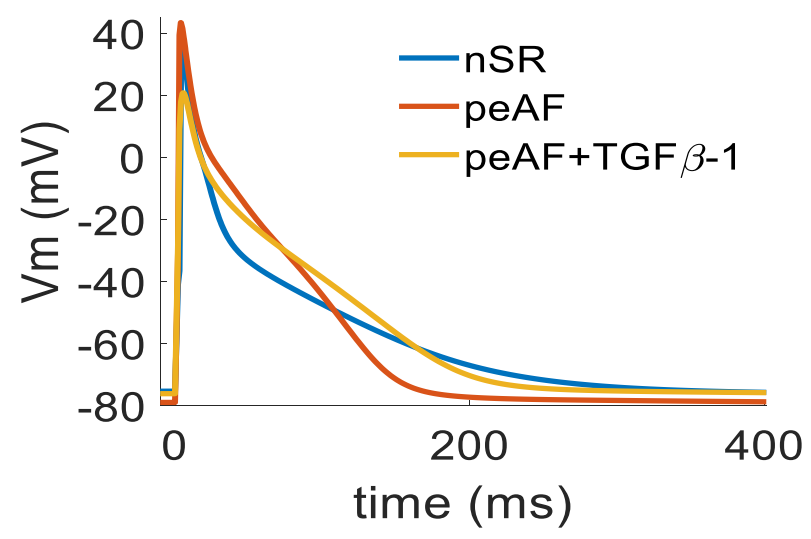

Figure 2. AP of a single myocyte. The blue line represents an AP under physiological conditions (nSR). The red line shows an AP under electrical remodeling (peAF) and the yellow line shows the AP under the effect of electrical remodeling and paracrine effect of transforming growth factor beta 1 (TGF- $\beta 1$ ).

When the fibrotic density was low (10\% and $20 \%)$, the excitation was able to propagate across the fibrotic region. In contrast, for higher density (40\%), conduction was blocked in this region. Reentry changed gradually from functional to anatomical due to block of conduction.

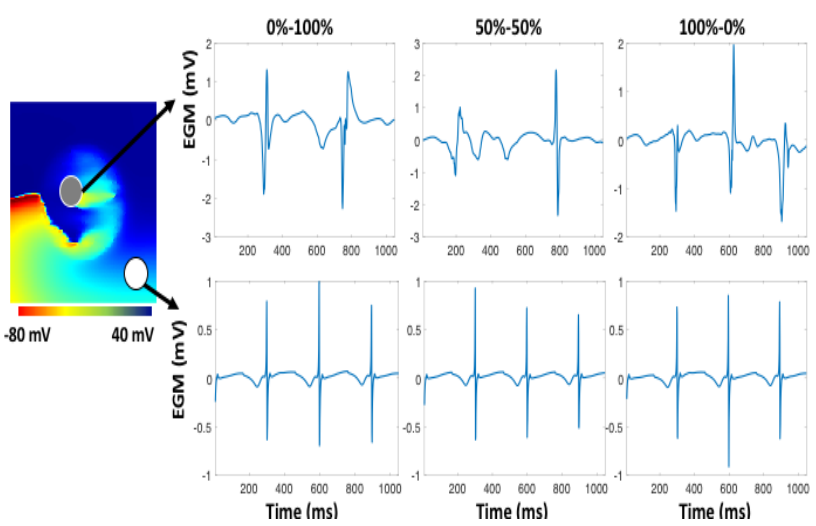

Figure 3. Bipolar EGM morphologies at three different points of the tissue with $10 \%$ of fibrosis density (white and gray dots indicate the location in the tissue). Depending on the myofibroblasts-collagen ratios, EGMs exhibited different morphologies.

EGMs in Figure 3 shows the different morphologies inside and outside of the fibrotic region. Depending on the myofibroblast-collagen ratio EGM's morphology as also affected. Additionally, the EGM morphology outside of the fibrotic did not change significantly.

EGM activity duration increased inside fibrotic region compared to the duration from the outside of this region. For fibrotic density of $10 \%$ we detected segments with shorter duration $(45.21 \pm 1.24 \mathrm{~ms})$. Increasing fibrosis density $(20 \%)$ increased the duration of the segments $(60.73 \pm 1.10 \mathrm{~ms})$, and for higher density of fibrosis $(40 \%)$ we detected segments with duration of $(46.59 \pm 1.29 \mathrm{~ms})$ or inactivity near the core. Figure 4 shows the difference in duration of the active segments and Shannon Entropy found inside and outside of the fibrotic region.

Fibrotic regions with lower percentage of myofibroblasts (below 50\% compared to the percentage of collagen), had segments with shorter duration. When increasing the percentage of myofibroblast densities (above 50\% compared to the percentage of collagen), propagation exhibited a slower frequency of activation which is reflected in segments with longer duration of activity.

For simulations with $40 \%$ fibrotic density, we were not able to detect any activity near the core. This center of the fibrotic region had an EGM with smaller peak to peak amplitude (below $0.5 \mathrm{mV}$ ). However, the Shannon entropy was homogenously distributed and higher compared to the values from outside the fibrotic region.

\section{Discussion}

Heterogeneities in the fibrotic tissue do not only alter the dynamics of the reentrant activity but can also change the morphology of the EGM.

Ashihara et al. [12] showed that fibroblasts present in the atrial substrate can alter the morphology of the EGM. Our results also show that myofibroblasts changed the morphology of the EGM. Since myofibroblasts tend to homogenize the cardiac tissue, segments with shorter activity duration were found in comparison with simulations where only collagen was present.

Our simulations showed that the modeling methodology clearly affects the dynamics of the reentry. Roney et al. [13] also showed that modeling methodologies of fibrotic tissue have a large effect on rotor dynamics in 3D simulations. However, it is still not clear how fibrosis structure and transmurality may affect the morphology of the EGM. This will be subject for further investigation.

Understanding the mechanisms responsible for initiation, progression, and resolution of cardiac fibrosis is crucial and may help to design anti-fibrotic treatment strategies and ablation therapies for patients with peAF.

\section{Conclusions}

In conclusion, fibrosis densities and its arrangement can alter the dynamics of the reentry and the morphology of EGMs. Higher myofibroblasts vs. collagen ratios in the fibrotic region leads to activity with longer duration. 


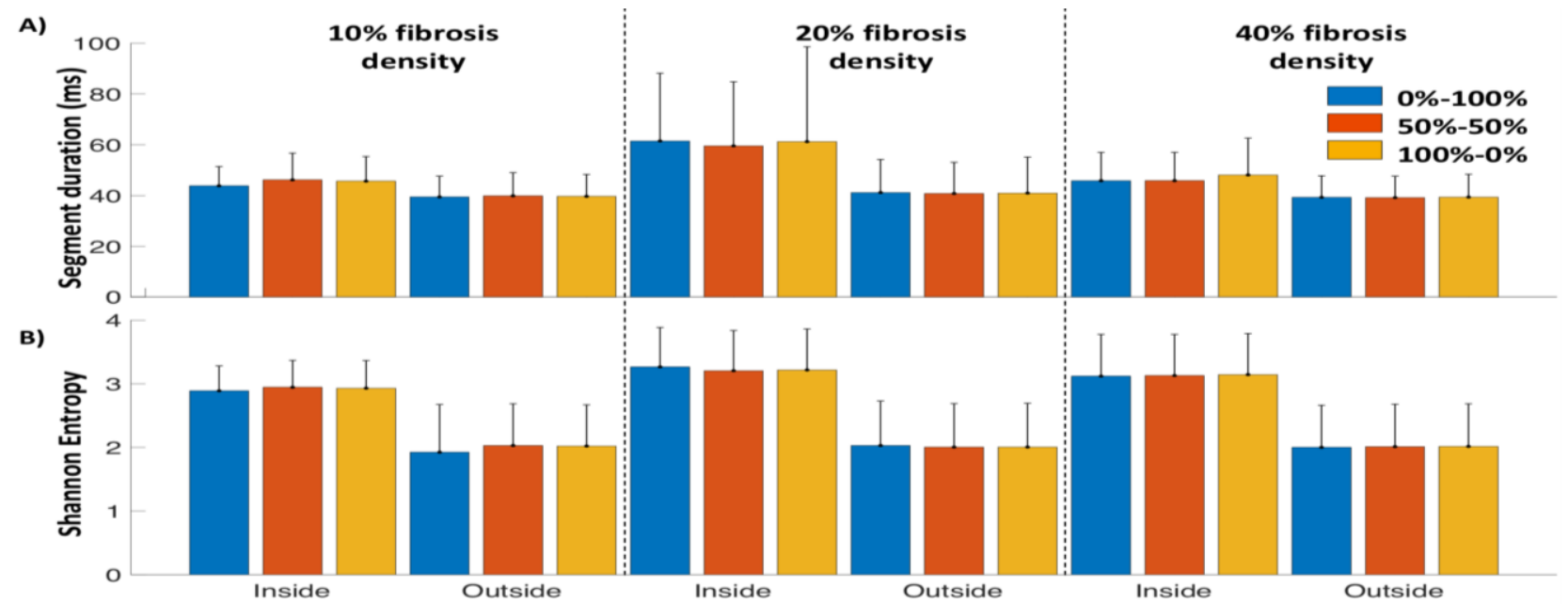

Figure 3. A) Mean duration of the active segment for different fibrosis densities and myofibroblasts vs. collagen ratios inside and outside the fibrotic region. As fibrosis density increases, the duration of the detected segment also increases. At 40\% fibrosis density voltage becomes very small and block can occur. B) Mean Shannon Entropy for inside and outside of the fibrotic region. Different densities of fibrosis increase the Shannon Entropy, it is not significantly affected by the ratio of myofibroblasts vs. collagen.

\section{Acknowledgments}

We gratefully acknowledge financial support by Deutsche Forschungsgemeinschaft (DFG) under grant LO 2093/1-1.

\section{References}

[1] K. S. McDowell et al., "Mechanistic inquiry into the role of tissue remodeling in fibrotic lesions in human atrial fibrillation," Biophys. J., vol. 104, no. 12, pp. 2764-2773, 2013.

[2] S. Zahid et al., "Patient-derived models link re-entrant driver localization in atrial fibrillation to fibrosis spatial pattern," Cardiovasc. Res., vol. 110, no. 3, pp. 443454, 2016.

[3] R. Morgan, M. A. Colman, H. Chubb, G. Seemann, and O. V. Aslanidi, "Slow conduction in the border zones of patchy fibrosis stabilizes the drivers for atrial fibrillation: Insights from multi-scale human atrial modeling," Front. Physiol., vol. 7, no. OCT, pp. 1-15, 2016.

[4] J. T. Koivumäki, T. Korhonen, and P. Tavi, "Impact of sarcoplasmic reticulum calcium release on calcium dynamics and action potential morphology in human atrial myocytes: A computational study," PLoS Comput. Biol., vol. 7, no. 1, 2011.

[5] A. Ferrer et al., "Detailed anatomical and electrophysiological models of human atria and torso for the simulation of atrial activation," PLoS One, vol. 10, no. 11, pp. 1-29, 2015.

[6] J. T. Koivumäki, G. Seemann, M. M. Maleckar, and P. Tavi, "In Silico Screening of the Key Cellular Remodeling Targets in Chronic Atrial Fibrillation," PLoS Comput. Biol., vol. 10, no. 5, 2014.
[7] J. Koivumäki et al., "Na+ current expression in human atrial myofibroblasts: Identity and functional roles," Front. Physiol., vol. 5 JUL, no. August, pp. 1-14, 2014.

[8] C. Poulet, S. Künzel, E. Büttner, D. Lindner, D. Westermann, and U. Ravens, "Altered physiological functions and ion currents in atrial fibroblasts from patients with chronic atrial fibrillation.," Physiol. Rep., vol. 4, no. 2, p. e12681, 2016

[9] N. Salvarani, A. Maguy, S. A. De Simone, M. Miragoli, F. Jousset, and S. Rohr, "TGF$\beta 1$ (Transforming Growth Factor- $\beta 1$ ) Plays a Pivotal Role in Cardiac Myofibroblast Arrhythmogenicity," Circ. Arrhythmia Electrophysiol., vol. 10, no. 5, 2017.

[10] M. M. Maleckar, J. L. Greenstein, W. R. Giles, and N. A. Trayanova, "Electrotonic coupling between human atrial myocytes and fibroblasts alters myocyte excitability and repolarization," Biophys. J., vol. 97, no. 8, pp. 2179-2190, 2009.

[11] C. Schilling, M. P. Nguyen, A. Luik, C. Schmitt, and O. Dössel, "Non-linear energy operator for the analysis of intracardial electrograms," IFMBE Proc., vol. 25, no. 4, pp. 872-875, 2009.

[12] T. Ashihara et al., "The role of fibroblasts in complex fractionated electrograms during persistent/permanent atrial fibrillation: Implications for electrogram-based catheter ablation," Circ. Res., vol. 110, no. 2, pp. 275 284, 2012.

[13] C. H. Roney et al., "Modelling methodology of atrial fibrosis affects rotor dynamics and electrograms," $E P$ Eur., vol. 18, no. suppl_4, pp. iv146-iv155, Dec. 2016.

Address for correspondence:

Jorge Sánchez Arciniegas

Institute of Biomedical Engineering, Karlsruhe Institute of Technology (KIT), Kaiserstr. 12, 76131, Karlsruhe, Germany. publications@ibt.kit.edu 